\title{
Investigation of an Alternative Sulfonated Silane in Ceramic Carbon Electrodes for Fuel Cell Applications
}

\author{
O'Rian O. Reid, E. Bradley Easton* \\ Electrochemical Materials Lab, Faculty of Science, Ontario Tech University ${ }^{\text {, }}$ \\ 2000 Simcoe Street N, Oshawa, ON, Canada L1G 0C5 \\ *Corresponding author: E-mail: Brad.Easton@uoit.ca
}

\begin{abstract}
In this work the sulfonated CCE material developed by Eastcott and Easton was replaced with a more alternative sulfonated organosilane monomer component. Specifically, 3-(trihydroxysilyl) propane-1-sulfonic acid (TPS) was replaced with 4-(2-(trihydroxysilyl)ethyl) benzenesulfonic acid (TEBS) and compared to the previous TPS-based system as well as the traditional Nafion ionomer electrode. The sulfonated CCEs were characterized using BET surface area analysis, thermogravimetric analysis, SEM, and TEM. The electrodes were then evaluated a full cell environment. The TEBS-based electrode showed a decrease in performance at both high and low relative humidities compared to the TPS/TEOS and Nafion ionomers. Impedance studies revealed that water retention with the TEBS was significantly lower that TPS, which accounted for the lower performance.
\end{abstract}

Keywords: ceramic carbon electrode; fuel cells; catalyst layers; electrochemical impedance spectroscopy

\footnotetext{
${ }^{1}$ Formerly known as University of Ontario Institute of Technology
} 
O.O. Reid, E.B. Easton, ECSarXiv (2019). doi:10.1149/osf.io/9n4mz.

\section{INTRODUCTION}

A traditional fuel cell electrode is comprised of two components, the catalyst layer (CL) that contains the carbon support, the metal catalyst, and the ionomer and the diffusion layer (GDL). These components allow the interaction of protons, electrons, and gases through contact at the catalyst site. In order for the oxygen reduction reaction (ORR) to take place a three-phase boundary has to be present where the ionomer is in contact with the carbon support and gas reactants are able to diffuse to the catalyst site $[1,2]$. The GDL allows the diffusion of reactant gas to the electrode and is typically made up of fibrous carbon that can be coated with polytetrafluoroethylene (PTFE) to prevent water from blocking gas diffusion. The carbon support is typically made up of a high surface area carbon material such as Vulcan carbon. The high surface area allows a larger dispersion of the metal catalyst used, and at the same time allows the transport of electrons to and from the catalyst sites and GDL. A microporous layer (MPL) is added between the GDL and CL and contains a colloidal dispersion of carbon black and PTFE, which not only enhances surface roughness, but also aids in the maintenance of water during fuel cell operation $[3]$.

Currently, platinum has been proven to be the best catalyst for the ORR [2]. The use of platinum accounts for a large portion of the fuel cell cost [2], but is the only metal that is efficient enough to be used in the slow kinetics of the ORR which is the rate limiting step of the water forming process.

The ionomer is another important component of the CL as it allows the transport of ions from the catalyst sites to the membrane in the anode, and from the membrane to the catalyst sites in the cathode. The ionomer also acts as a binding agent and allows better contact with the catalyst sites in order to have a three-phase boundary where a proton, electron, and reactant gas are present for the ORR to take place. As a result the performance of the fuel cell increases substantially and 
aids in the increase of platinum utilization in the CL [1]. The ionomer has to be able to mix well with the membrane to facilitate good adhesion, making Nafion the most common ionomer used in the CL. Nafion brings great benefits that are perfect for the catalyst layer, but is also costly and as mentioned water is vital for the transport of protons resulting in poor transport in low relative humidity $(\mathrm{RH})$ conditions. The addition of Nafion can also be detrimental to the electrode performance if too much is added especially if the quantity added covers catalyst sites. As a result, a loading of $30 \mathrm{wt} \%$ Nafion is typically used since it has been proven to be the quantity required to give the best performance when a $20 \% \mathrm{Pt} /$ Vulcan type catalyst is used [4, 5]. The use of various sulfonated hydrocarbon polyelectrolytes including sPEEK, sulfonated polyethersulfone, and sulfonated polyetherimide have been studied in an attempt to decrease the cost of the fuel cell and increase the water retention in low RH environments[6]. Compared to Nafion these hydrocarbons based polyaromatics are less expensive, can easily be functionalized to increase proton conductivity, and have high gas permeability which make them suitable candidates for ionomers[68]. The downfall of these ionomer materials is their sensitivity to changes in RH, just like with Nafion, and as a result exhibit a decrease in performance. The incorporation of these alternative ionomer materials with the carbon supported catalyst is also an issue, especially if there is limited contact preventing interactions between protons, electrons, and gas reactants and decreasing the three-phase boundaries.

One type of electrode structure that has been studied over recent years is the ceramic carbon electrode (CCE). A CCE is an electrode containing a carbon support bound together with a silica polymer. This type of electrode provides even dispersion of a carbon support, and through the addition of hydrophilic groups such as sulfonic acid, the conductivity of ions and manipulation of water absorption and retention can be possible [9]. Through their synthesis using the sol-gel method, the nature of the CCE can also be altered to give a very porous material which can increase 
the number of catalyst sites available for three-phase boundaries. CCEs have been studied extensively in the use of a modified surface polymer electrodes to replace carbon paste electrodes by Lev and co-workers. They found that replacing a carbon paste electrode with a CCE shows an increase in the signal to noise ratio, as well as better stability of the electrode over numerous cycles [10]. Ranganathan and Easton have also shown the use of CCEs in electrolysis cells in the $\mathrm{CuCl}$ thermochemical cycle to enhance the crossover of anionic species [11].

Eastcott and Easton have modified the CCE preparation method for the use in fuel cell electrodes. In their work the carbon supported catalyst was mixed with 3-(trihydroxysilyl)-1propane sulfonic acid (TPS) and tetraethyl orthosilicate (TEOS). This colloidal mixture was then spray deposited on a GDL and used as the cathode of a $\mathrm{H}_{2} / \mathrm{O}_{2}$ fuel cell, where an increase surface area, as well as consistent fuel cell performance at low RHs was observed[12]. This improvement to the electrode by the addition of an organosilane ionomer shows great promise for automotive applications since it does not demonstrate sensitivity to changes in RH environments, while their preparation through the sol-gel chemistry allows different CCE materials to be prepared for a variety of fuel cell applications.

One possible ion conducting monomer that could show increased performance at multiple RH conditions is 4-(2-(trihydroxysilyl)ethyl) benzenesulfonic acid (TEBS). This monomer has been proven to increase the ionic conductivity for a number of applications both in the ionomer and the membrane. The exploration of using TEBS exclusively as an ionomer material to increase ion conductivity in the electrodes is non-existent, however, the use of TEBS as other components have been studied. De Almeida et al. used TEBS as a dopant in Nafion membranes with various loadings and the proton conductivity was measured. The doping of Nafion membranes with TEBS demonstrated an increase in proton conductivity and water retention in low RH conditions with low TEBS loadings, but as the loading increased conductivities similar to pure Nafion were 
observed [13]. Easton and Pickup also used TEBS in an attempt to decrease the Nafion content by functionalizing the carbon support with a monolayer of the TEBS monomer. When treated with TEBS, it was determined that a lower Nafion loading was required to observe similar results to traditional fuel cell MEAs [5].

In this work we prepared sulfonated CCEs using TPS and TEBS and compared how PEMFC performance is affected when a different sulfonated silane is used. The CCEs studied using the same ratio of sulfonated to unsulfonated silane that was used by Eastcott et. al. The sulfonated CCE materials will be tested on the cathode side of a full fuel cell with a standard $20 \%$ $\mathrm{Pt} / \mathrm{C}$ catalyst and Nafion as the ionomer on the anode. Electrode performance was assessed at various cathode $\mathrm{RH}$ values in order to evaluate differences in water retention. Cyclic voltammetry (CV) and electrochemical impedance spectroscopy (EIS) was also performed in order to understand how water management is affected when an alternative sulfonated precursor was used.

\section{EXPERIMENTAL}

\subsection{Electrode PreParation}

\subsubsection{CCE Preparation}

The preparation of CCE materials was completed by following the method described in our previous publications [14-18]. Briefly, CCEs were prepared using 2 different sulfonated silane precursors 4-(2-(trichlorosilyl)ethyl)benzenesulfonyl chloride (TCBS, Gelest, 50\% in dichloromethane) which upon hydration TCBS is converted to 4-(2-(trihydroxysilyl)ethyl) benzenesulfonic acid (TEBS), or 3-(trihydroxysilyl)-1-propane sulfonic acid (TPS, Gelest, 30-35 wt $\%$ in $\mathrm{H}_{2} \mathrm{O}$ ). $20 \% \mathrm{Pt}$ on Vulcan $\mathrm{XC} 72$ carbon black (Premetek) weighed in a clean, dry glass beaker. Deionized water was then added to the beaker to prevent ignition of platinum upon the addition of methanol. The colloidal mixture was stirred mechanically and the addition of methanol 
(Fisher) was made. The sulfonated silane and tetraethylorthosilicate (TEOS, Sigma) were added drop-wise such that the atomic ratio of sulfonated silane-to-TEOS was 5:95 and the $\mathrm{wt} \%$ of silicate in the final $\mathrm{CCE}$ was ca. $40 \mathrm{wt} \%[14,17] . \mathrm{NH}_{4} \mathrm{OH}$ (Sigma) was added to ensure the reaction proceeded under slightly basic conditions. The mixture and set to stir for ca. 72 hours after which the partially gelled solution was spray deposited onto the gas diffusion layer (GDL). In all cases, the GDL used on wet-proofed carbon fibre paper (Toray TPGH-090, $10 \mathrm{wt} \%$ wet-proofing) onto which a microporous layer (MPL) of carbon of Teflon was previously applied [19]. Following deposition, the resulting electrodes was dried for $30 \mathrm{~min}$ at both room temperature and $135^{\circ} \mathrm{C}$. Each CCE had a platinum loading of $0.45 \pm 0.05 \mathrm{mg} \mathrm{cm}^{-2}$

\subsubsection{Nafion-based Electrodes}

Nafion based catalyst layers (CL) were prepared for testing standard materials to compare to CCE materials. To produce Nafion based CL's a similar method to CCE fuel cell catalyst layer preparation was used. In this case $20 \%$ Pt on Vulcan XC72 carbon black (Premetek) was mixed with deionized water and isopropyl alcohol in a 50:50 ratio, after which the appropriate volume of $5 \mathrm{wt} \%$ Nafion (Ion Power Inc., $5 \mathrm{wt} \%$ in aliphatic alcohols and water) was added to give a Nafion loading of $30 \mathrm{wt} \%$. The mixture was then sonicated for 1 hour and set to stir overnight before spraying on wet-proofed carbon fibre paper (Toray TPGH-090, $10 \mathrm{wt} \%$ wet-proofing). The resulting electrodes then dried for 30 min at both room temperature and $135^{\circ} \mathrm{C}$. A platinum loading of $0.41 \pm 0.03 \mathrm{mg} \mathrm{cm}^{-2}$ was attained. The Nafion based electrode will hereafter be referred to as PE20.

\subsection{MEA PREPARATION}

Membrane electrode assemblies (MEA) were prepared by cutting a $5 \mathrm{~cm}^{2}$ square of the Nafion based P20 electrode which was used as the anode for all fuel cell tests. A $5 \mathrm{~cm}^{2}$ square of 
either one of the CCEs or another P20 electrode was used as the cathode. The two electrodes were placed facing each other with a Nafion NRE 212 membrane (Ion Power) in the middle. The MEA was placed between two sheets of aluminum foil and then between two aluminum plates and hotpressed at $150 \mathrm{~kg} \mathrm{~cm}^{-2}$ at $130^{\circ} \mathrm{C}$ for 180 seconds using a Carver laboratory press. The MEA was removed from the press and placed inside the fuel cell test fixture.

\subsection{Fuel Cell Testing And Electrochemical MEASurements}

Each MEA was tested in a $5 \mathrm{~cm}^{2}$ test fuel cell test fixture (Fuel Cell Technologies) connected to a commercial fuel cell test station (Fuel Cell Technologies, 120A unit) controlled using Labview software. This station allowed the control of gas flow rates, gas back pressure, humidifier temperatures and potential load. Data was acquired at a cell temperature of $80{ }^{\circ} \mathrm{C}$ and with 10 psig $(170 \mathrm{kPa})$ back pressure at the anode and cathode. Reactant gases were humidified by passing them through the stations humidifier bottles before reaching the fuel cell. $\mathrm{O}_{2}$ gas or air, depending on the test being conducted, were passed through the cathode at a flow rate of $200 \mathrm{~mL}$ $\mathrm{s}^{-1}$ and $1000 \mathrm{~mL} \mathrm{~s}^{-1}$ respectively, and $\mathrm{H}_{2}$ gas was passed through the anode at a flow rate of 100 $\mathrm{mL} \mathrm{s}^{-1}$. The relative humidity (RH) levels of the flowing gasses were controlled by varying the humidifier temperature with respect to the cell temperature. $\mathrm{H}_{2}$ gas was passed through the anode and was heated at $80{ }^{\circ} \mathrm{C}$ to allow $100 \% \mathrm{RH}$ in the anode while $\mathrm{O}_{2}$ and air were passed through the cathode humidifiers which were heated to temperatures between $80^{\circ} \mathrm{C}$ and $45^{\circ} \mathrm{C}$ in order to obtain relative humidities between $100 \%$ and $20 \%$.

Cyclic voltammetry (CV) and electrochemical impedance spectroscopy (EIS) measurements were conducted using a Solartron 1470E multichannel potentiostat paired with a Solartron 1260 frequency response analyzer controlled using Multistat software (Scribner 
Associates). CV measurements were performed for a potential range between 0.08 and $1.4 \mathrm{~V}$ (vs RHE) with humidified $\mathrm{N}_{2}$ flowing at the cathode and with the $\mathrm{H}_{2}$ anode serving as both the reference and the counter electrode. CV's were collected at a cell temperature of $30{ }^{\circ} \mathrm{C}$ at $100 \%$ RH using a sweep rate of $50 \mathrm{mV} \mathrm{s}^{-1}$. The electrochemically active surface area (ECSA) of platinum in the electrode was determined from the area under the anodic sweep in the HupD region of the $\mathrm{CV}\left(1 \mathrm{~cm}^{2} \mathrm{Pt}_{\mathrm{t}}=0.210 \mathrm{mC}\right)$.

EIS measurements were collected over a frequency range of $100 \mathrm{kHz}$ to $0.1 \mathrm{~Hz}$ at a DC bias potential of $0.425 \mathrm{~V}$ (vs. RHE). EIS data was analyzed using a finite transmission-line model [20-24]. EIS measurements were performed with humidified $\mathrm{N}_{2}$ flowing at the cathode and with the $\mathrm{H}_{2}$ anode serving as both the reference and the counter electrode. Measurements were made at cell temperatures of both $30{ }^{\circ} \mathrm{C}$ and $80{ }^{\circ} \mathrm{C}$, with $100 \% \mathrm{RH}$ at the anode and variable levels of RH at the cathode.

\subsection{MAterials CHARACTERIZATION}

Transmission Electron Microscopy (TEM) images were of CCE materials were collected using a Zeiss Libra $200 \mathrm{MC}$ microscope operated at $200 \mathrm{kV}$ located at the the University of Waterloo (WATLab). Thermogravimetric analysis (TGA) information was collected using a TA Instruments Q600 SDT thermal analyzer. Samples were heated from room temperature to $1000{ }^{\circ} \mathrm{C}$ at a rate of $20^{\circ} \mathrm{C} \mathrm{min}^{-1}$ under flowing air in order to determine the weight percent of the electrode components. Brunauer-Emmett-Teller (BET) surface area measurements were performed using a Quantachrome NOVA 1200e Surface Area and Pore Size Analyzer. 


\section{RESUltS AND DiscusSion}

\subsection{Physical Properties of CCES}

TEM images were collected on all electrode materials in order examine the silicate distribution at the nanoscale and are shown in Figure 1. The Premetek 20\% Pt/C catalyst material has the expected distribution of Pt nanoparticles dispersed onto the carbon black support. The average particle size of platinum was measured to be ca. $4 \mathrm{~nm}$. This structure was retained in both CCE compositions. While there was no obvious signs of silicate agglomeration, the CCE compositions showed less dispersion on the TEM grid, indicating that the silicate is serving to bind these catalyst particles, serving as a "nanoglue" as commonly described in Rolison's CCE work $[25,26]$. Based on this, one would not expect the presence of the silicate to impede ion or electron transport to and from the catalyst surface.

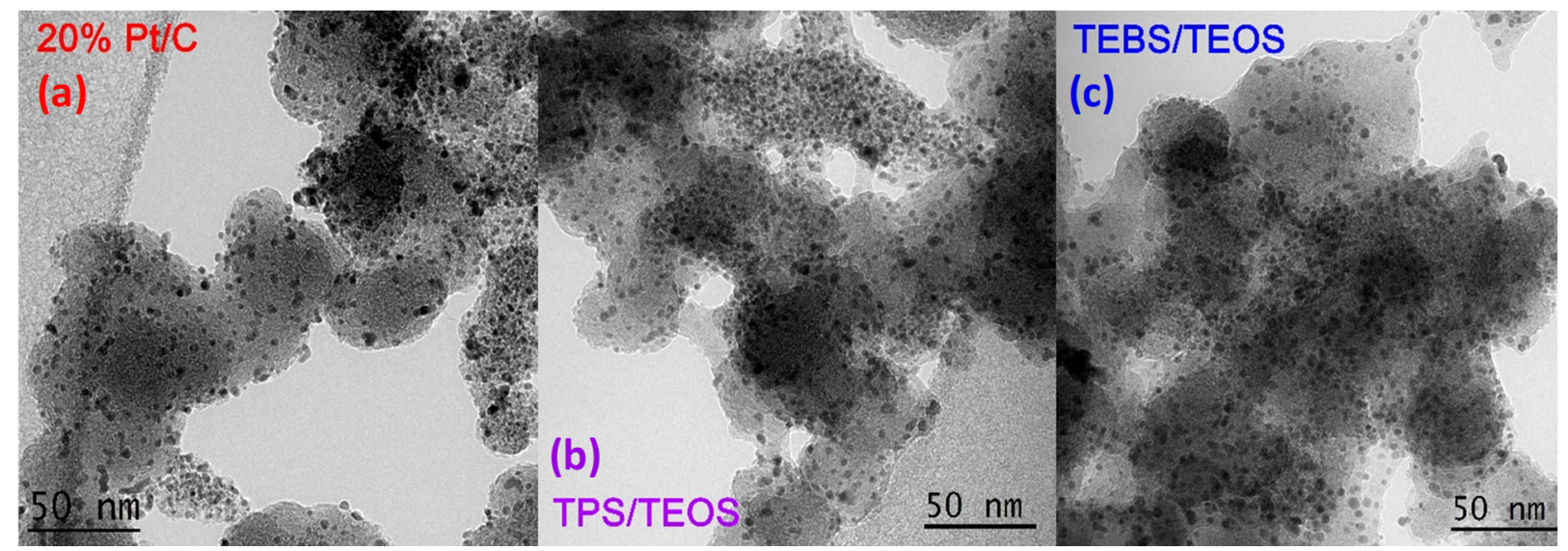

Figure 1: TEM images obtained for (a) $20 \% \mathrm{Pt} / \mathrm{C}$ (b) the TPS-based CCE, and (c) the TEBSbased CCE.

TGA analysis was used to determine compositional information on the electrode materials.

Figure 2 illustrates the TG and DTG curves obtained for the $20 \% \mathrm{Pt} / \mathrm{C}$ and the two sulfonated CCE materials in flowing air. All samples displayed a large mass loss associated with the combustion of carbon (to $\left.\mathrm{CO}_{2}\right)$. The base catalyst material $(20 \% \mathrm{Pt} / \mathrm{C})$ began to combust at ca. $400{ }^{\circ} \mathrm{C}$ which completed at ca. $500{ }^{\circ} \mathrm{C}$, leaving a residual mass of $20 \mathrm{wt} \%$ due to the Pt. This carbon combustion 
was observed for each CCE material, though it occurred at slightly higher temperatures (470 - 550 $\left.{ }^{\circ} \mathrm{C}\right)$ compared to the base catalyst. This is attributed to a thin layer of silicate surrounding the carbon particles, likely bound to the surface of the carbon through surface hydroxyl groups [27], effectively protecting it from combusting at lower temperatures. A similar phenomena was also observed by Atif et al. for carbon blacks modified with silanes [28]. The TPS-based material exhibited a small mass loss around $200^{\circ} \mathrm{C}$ which is associated with the loss of sulfonic acid groups[29]. This loss is ca. $3 \mathrm{wt} \%$ of the sample which is close to the value observed by Eastcott et.al [17]. This is also seen in the TEBS-based material with a $3 \%$ loss between $200^{\circ} \mathrm{C}$ and $400^{\circ} \mathrm{C}$ caused by the degradation on the ethylphenyl sulfonic acid group. The final mass for both CCE materials is also higher and is caused by the residual $\mathrm{SiO}_{2} / \mathrm{SiO}_{1.5}$ network as well as the platinum from the $\mathrm{Pt} / \mathrm{C}$ catalyst.

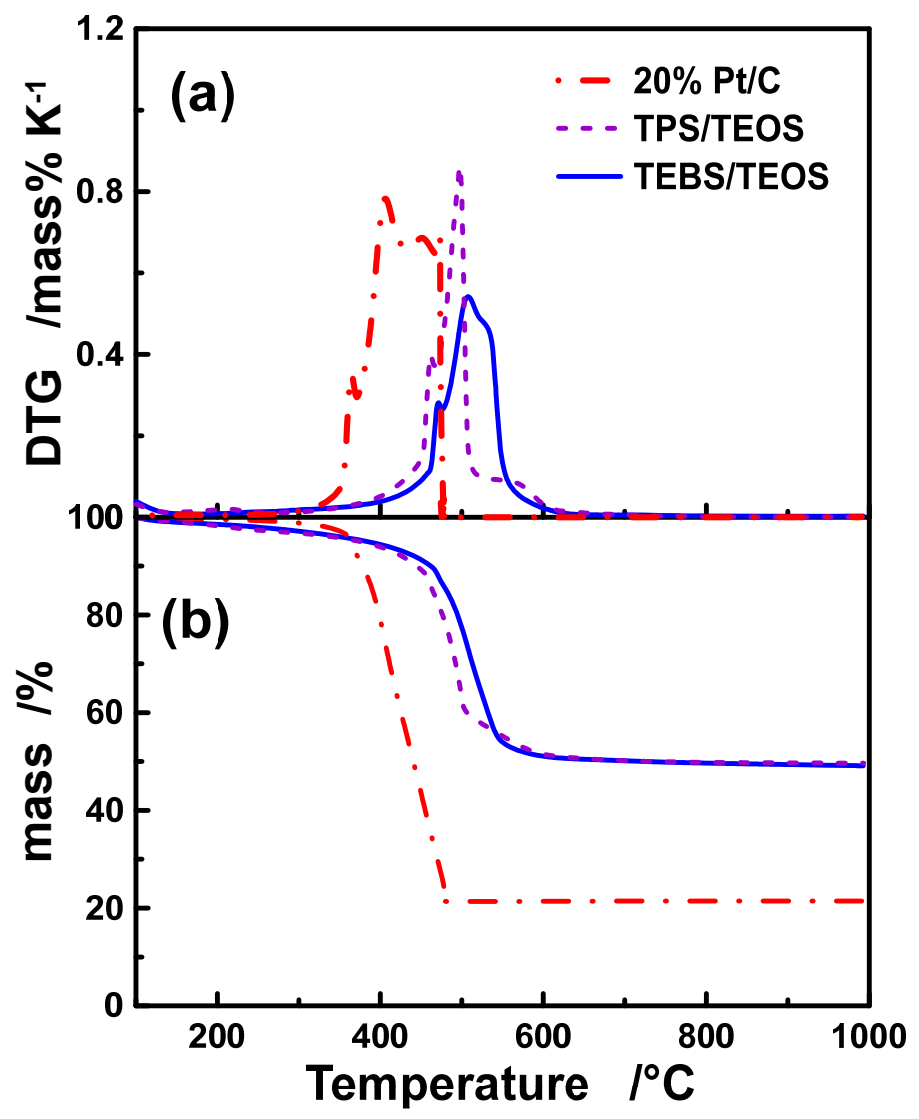

Figure 2: Comparison of a) TGA and b) DTG plots in the presence of air at $20{ }^{\circ} \mathrm{C} \mathrm{min}-1$ for $20 \% \mathrm{Pt} / \mathrm{C}$, TPS-based, and TEBS-based materials. For these plots, not all points are indicated. 
The BET surface area was measured for each of the CCE materials, the $20 \% \mathrm{Pt} / \mathrm{C}$ and the Vulcan XC72 carbon black support, which are compiled in Table 1. The BET surface areas measured for both Vulcan XC72R and 20\% Pt/C were in close agreement with measured values in the literature [30, 31]. The carbon supported catalyst was expected to have a lower BET surface area than the bare carbon due to the mass of platinum on the surface. The TPS-based CCE demonstrated a larger surface area compared to the carbon supported catalyst, which can be attributed to the formation of smaller pores in the silicate network. The largest BET surface area was achieved with the TEBS-based CCE. This larger surface area is perhaps due to higher porosity in the silica/ionomer network.

Table 1: BET surface area of the CCE samples, the 20\% Pt/C, and Vulcan XC72R carbon support.

\begin{tabular}{|l|l|}
\hline Sample & BET Surface Area $/ \mathrm{m}^{2} \mathrm{~g}^{-1}$ \\
\hline Vulcan XC72R & 234 \\
\hline 20\% Pt/Vulcan (Premetek) & 190 \\
\hline TPS-CCE & 220 \\
\hline TEBS-CCE & 338 \\
\hline
\end{tabular}

\subsection{ELECTROCHEMICAL TESTING}

Prior to performance testing, $\mathrm{CV}$ measurements were performed at $30{ }^{\circ} \mathrm{C}$ to determine the ECSA of Pt each electrode in an MEA configuration. These CVs and the corresponding ECSA values are shown in Figure 3. All CVs demonstrated the typical platinum shape, with the TPSbased CCE showing slightly broader peak features. While all electrodes had ECSA values in the expected range for this catalyst, both CCEs had higher ECSA values than the Nafion-based electrode. This indicates that the organosilicate creates an electrode structure that access to Pt sites. This could partially be explained by the larger increased BET surface in the CCEs. However, maximum ECSA was achieved with TPS-based CCE which had a lower BET surface area than the TEBS-based electrode. This, coupled with the fact that a larger double layer capacitance was 
observed with the TPS-based CCE, indicates that its pore structure creates a larger catalyst/ionomer interface than the other electrodes.
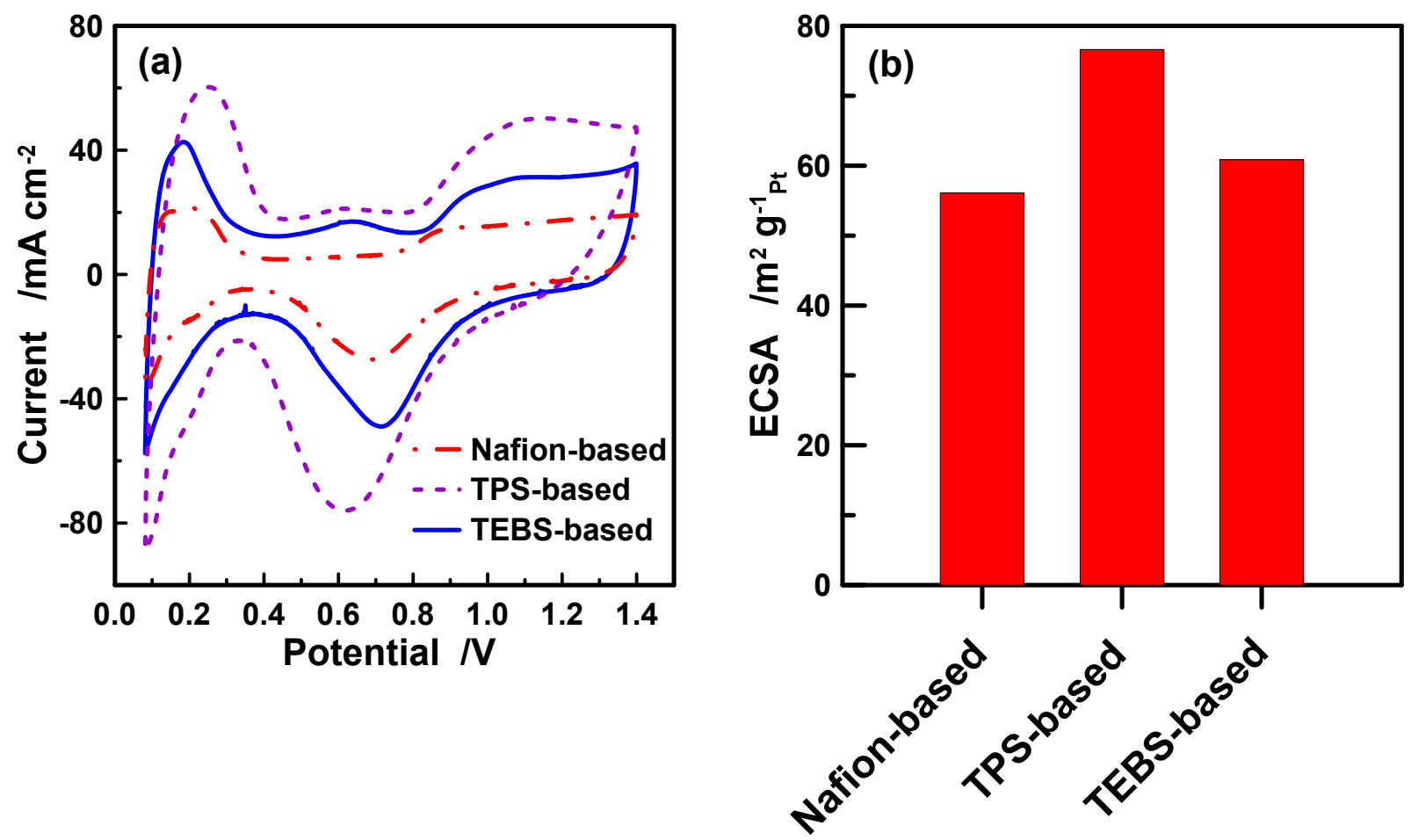

Figure 3: (a) CVs obtained for the Nafion-based, TPS-based, and TEBS-based ionomer electrode. (b) Comparison of the ECSA of each electrode. Measurements were made at $30{ }^{\circ} \mathrm{C}$ using a sweep rate of $100 \mathrm{mV} \mathrm{s}^{-1}$. Humidified $\mathrm{H}_{2}$ gas was supplied at the anode at a flow rate of $100 \mathrm{~mL} \mathrm{~min}^{-1}$ while humidified $\mathrm{N}_{2}$ was supplied to the cathode at a flow rate of $200 \mathrm{~mL} \mathrm{~min}^{-1}$.

To examine this further, EIS measurements were also performed at $30{ }^{\circ} \mathrm{C}$. Figure 4 compares the EIS response obtained with each MEA, shown as both Nyquist and capacitance plots. Figure 4d shows an expanded view of high frequency region of the Nyquist plots, where 45-degree Warburg responses are clearly visible for each sample. The projection of the Warburg length onto the real axis corresponds to $\mathrm{R}_{\Sigma} / 3$, where $\mathrm{R}_{\Sigma}$ is the total catalyst layer resistance which is composed of both ionic and electronic resistance [22]. The TPS-based electrode displayed the highest catalyst layer resistance, slightly larger than that measured for Nafion. Despite this larger resistance, the TPS-based electrode has the highest limiting (low frequency) capacitance, which is a measure of ECSA [20] and in agreement with the CV data. The lowest catalyst layer resistance was measured 
for the TEBS-based electrode, though it had a lower limiting capacitance than the TPS-based CCE. This indicates that the TEBS structure is a better proton conductor than the TPS structure, allowing faster exchange of ions with catalyst surface. However, this also indicates that the TEBS structure has a smaller interface with the $\mathrm{Pt} / \mathrm{C}$ surface, perhaps due to discrete regions or agglomerates of porous silicate that are not in contact with the $\mathrm{Pt} / \mathrm{C}$ surface. Nevertheless, both $\mathrm{CCE}$ compositions create electrode with Pt surface area and proton conductivity that are essentially as good as or better than that obtained with Nafion-based electrodes.
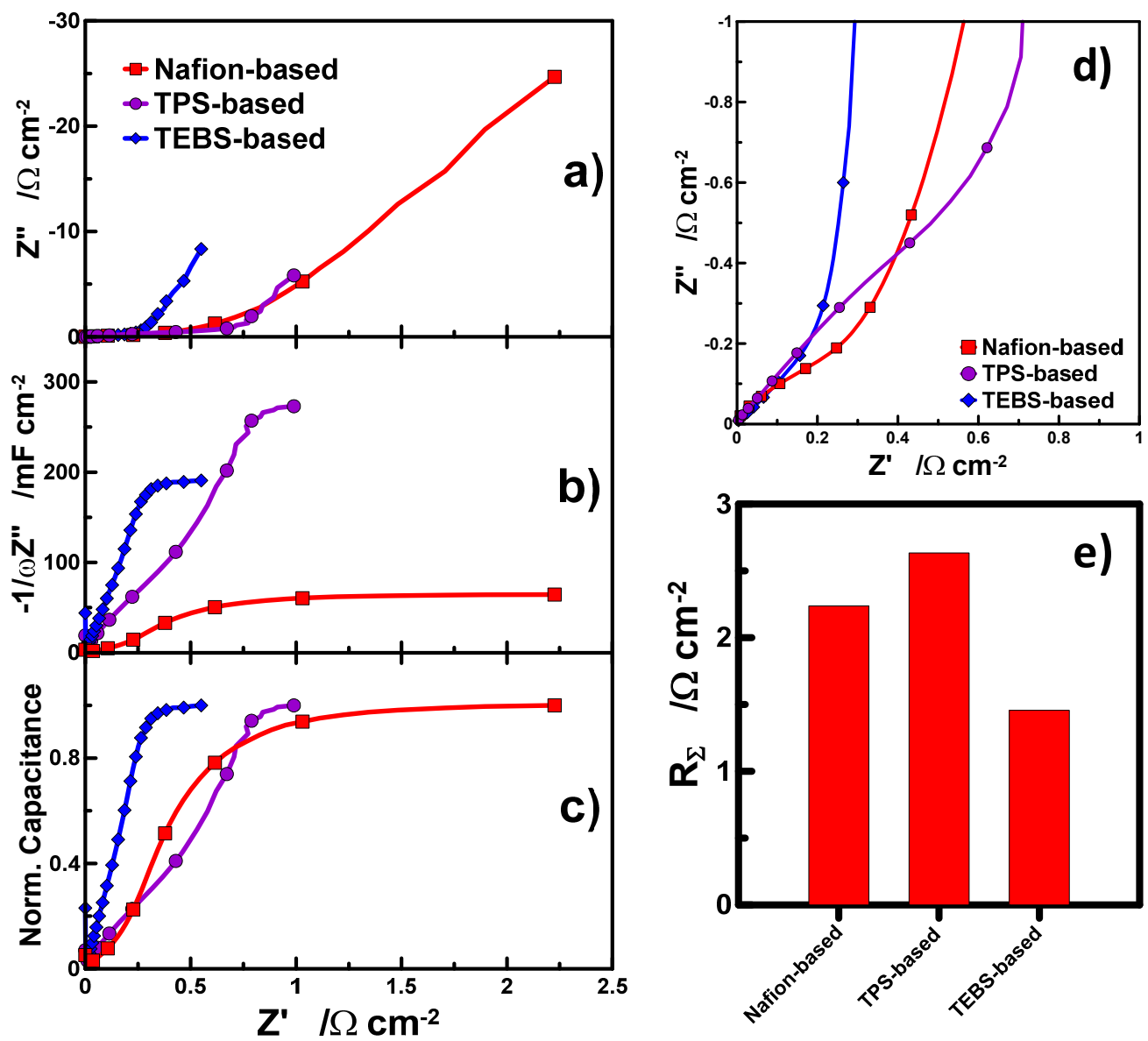

Figure 4: Comparison of a) Nyquist plot, b) Capacitance plot, c) Normalized capacitance plot obtained for MEAs prepared with Nafion-based, TPS-based, and TEBS-based cathodes. d) Expansion of the high frequency region of the Nyquist plot. e) Comparison of the total catalyst layer resistance, Rs, determined for each electrode. Measurements were made at $30{ }^{\circ} \mathrm{C}$ using a $\mathrm{DC}$ bias potential of $0.425 \mathrm{~V}$. Humidified $\mathrm{H}_{2}$ gas was supplied at the anode at a flow rate of 100 $\mathrm{mL}$ min $^{-1}$ while humidified $\mathrm{N}_{2}$ was supplied to the cathode at a flow rate of $200 \mathrm{~mL} \mathrm{~min}{ }^{-1}$. 
To examine if these properties translate into good fuel cell performance, each MEA was tested thoroughly at a cell temperature of $80^{\circ} \mathrm{C}$. It has been previously shown that CCEs are prone to flooding using high relative humidity $(\mathrm{RH})$ cathode gas feeds and actually perform better with drier gas feeds $[15,16]$. As such, all MEAs were tested using cathode gas feeds at multiple RH levels, varying from $20-100 \%$. Figure 5 compares polarization curves under the condition that correspond the relative humidity was tested a of the cathode gas feed was manipulated. The Nafion-based cathode showed limitations due to mass transport at $100 \% \mathrm{RH}$. While this was alleviated when the RH was decreased, ohmic losses increased sharply.
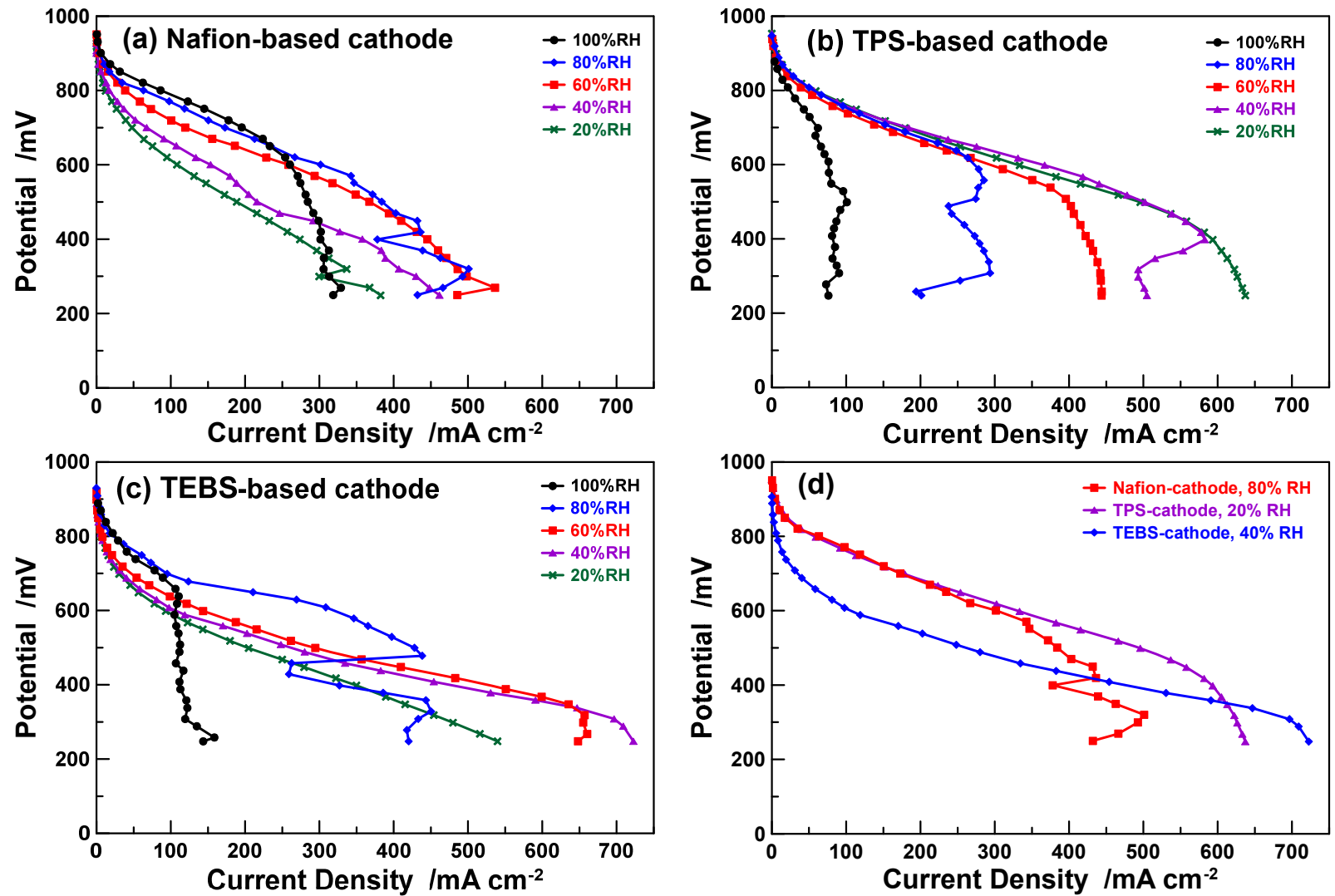

Figure 5: Variation in the $\mathrm{H}_{2}$ /Air polarization curves obtained for MEAs prepare with the (a) Nafion-based cathode (b) TPS-based cathode and (c) TEBS-based cathode. Measurements were made at a cell temperature of $80^{\circ} \mathrm{C}$ with a constant anode gas feed $\mathrm{RH}$ of $100 \%$ and variable cathode gas feed RH as indicated. (d) Comparison of the best performance obtained with each MEA. 
O.O. Reid, E.B. Easton, ECSarXiv (2019). doi:10.1149/osf.io/9n4mz.

The TPS-based electrode did not show any increase in ohmic losses at low RH, which indicates sufficient ion transport even in dry conditions and demonstrates a hygroscopic material. Furthermore, mass transport losses were minimized at lower RH, consistent with the results of Eastcott [12].

Compared to the other two electrodes, the TEBS-based electrode showed noticeably lower performance in the activation region indicating slow reaction kinetics for the ORR. A larger drop in polarization was noticed after $80 \% \mathrm{RH}$, which could be due to fluctuations in the curve caused by mass transport losses. The limitations caused by mass transport were also noticeable at $100 \%$ $\mathrm{RH}$, but below $80 \% \mathrm{RH}$ mass transport limitations were not observed to the same extent as in oxygen.

EIS was conducted to get an understanding of how water was being managed with the change in RH for each electrode. The EIS data was compared between each electrode and is displayed in 6 . The Nyquist plots between each electrode illustrated similar trends with an increase in Warburg length as the RH was decreased but was not seen as extensive in both CCE samples. The comparison of capacitance depicts the largest capacitance at all RHs with the TPS-based electrode. The TEBS-based electrode also demonstrated a capacitance that was higher than the Nafion-based electrode, which was then reduced to a value that was comparable to the Nafionbased electrode at $20 \% \mathrm{RH}$. This indicates a larger decrease in water content with TEBS than with TPS further demonstrating the hygroscopic nature between the two organosilane ionomers. The normalized capacitance also indicates an increase in resistance as RH is decreased, which was gradual from $100 \%$ to $20 \%$ RH for both Nafion-based and TPS-based electrodes, but for the TEBSbased electrode the decrease was more abrupt between $40 \%$ and $20 \%$ RH. 


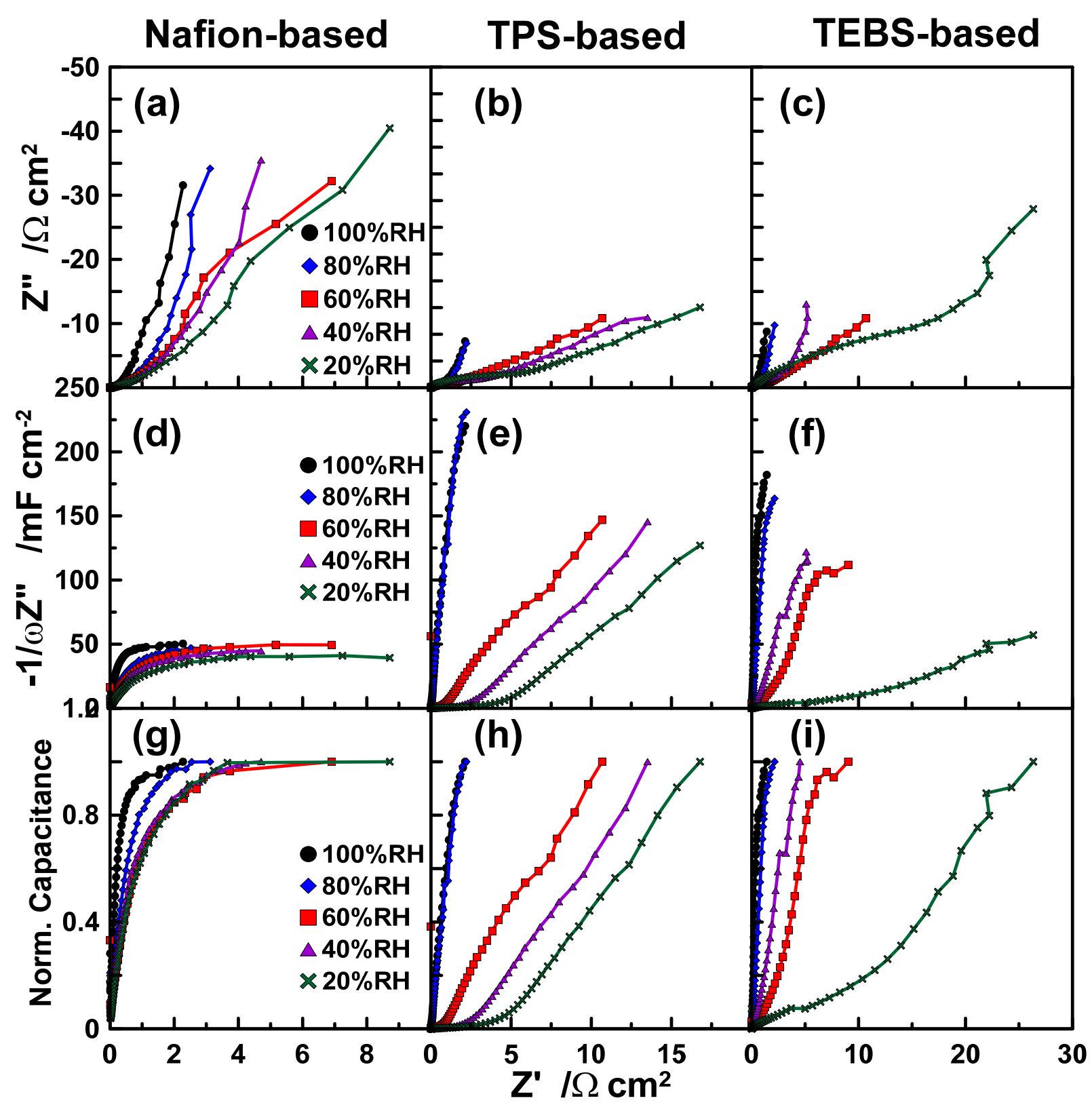

Figure 6: Variation in the EIS response with cathode gas feed in RH for the (a-c) Nafionbased, (d-f) TPS-based, and (g-i) TEBS-based electrodes. Measurements were made $80^{\circ} \mathrm{C}$ with a $\mathrm{H}_{2}$ anode feed and $\mathrm{N}_{2}$ cathode feed.

Figure 7 illustrates a comparison of the limiting capacitance, $\mathrm{R}_{\Sigma}$, and the membrane resistance as the RH is decreased. A decrease in capacitance was observed with both CCEs while the Nafion-based electrode stayed relatively the same. The limiting capacitance illustrated a larger decrease with the TEBS-based electrode when compared to the other two electrodes, most likely 


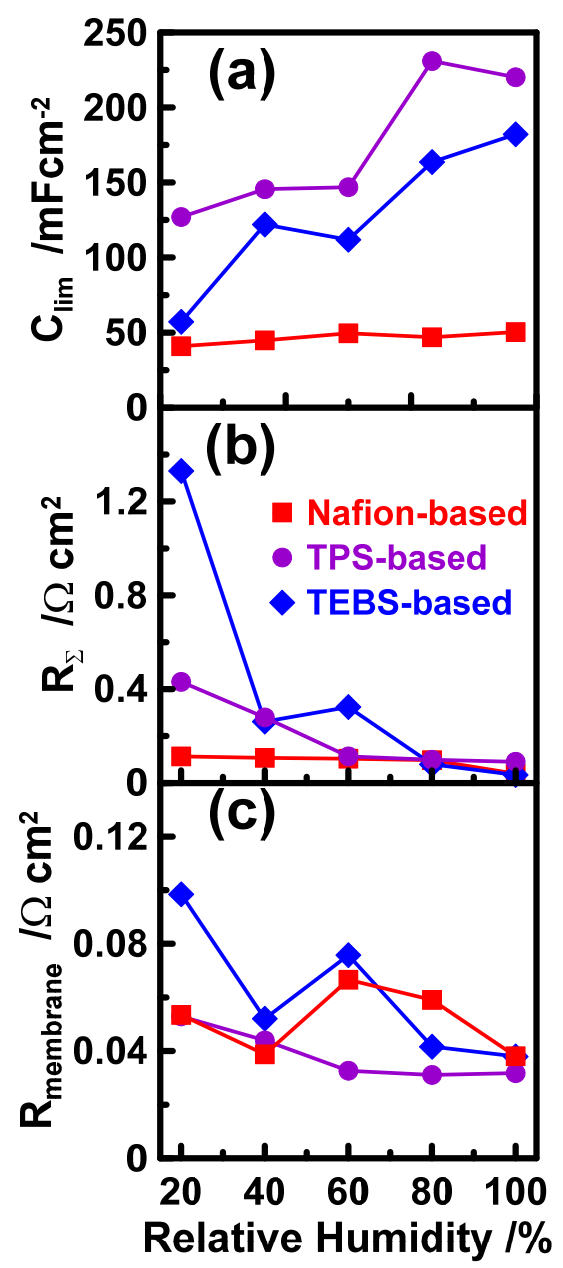

Figure 7: Comparison of a) limiting capacitance, $\mathrm{Clim}_{\mathrm{im}}$ b) total electrode resistance, and c) membrane resistance for the Nafion-based, TPS-based, and TEBS-based electrodes using variable cathode gas feed $\mathrm{RH}$ Measurements were made at a cell temperature of $80^{\circ} \mathrm{C}$ with a $\mathrm{H}_{2}$ anode feed and $\mathrm{N}_{2}$ cathode feed.

due to the lower hygroscopicity of the electrode which was also observed as an increase in ohmic resistance in the polarization curves. $\mathrm{R}_{\Sigma}$ showed an increase in both CCE electrodes with a decrease of RH, caused by hindered ion transport in the catalyst layer. This increase in resistance was more noticeable with the TEBS-based electrode, especially below $60 \% \mathrm{RH}$ which suggests a much lower water content in the electrode. This indicates that there is a larger $R_{\text {ionic }}$ contribution with TEBS when compared to the other electrodes. TPS-based electrode also demonstrates this, and when the Nafion-electrode is considered the difference in values are not as large. However, the membrane resistance for both CCE's initially demonstrated resistance values that were lower than the Nafion- 
based electrode. The electrode containing TPS shows a generally lower membrane resistance than the Nafion-based electrode. This trend was also observed by Eastcott et. al and is caused by the diffusion of liquid water from the highly hydrated cathode back to the drier anode, a phenomena that is commonly referred to as anode water removal (AWR) [12]. However, this was not observed to the same extent since the anode material was prepared in-house, and not commercially as was used by Eastcott et. al. This was not observed with TEBS, in fact the membrane resistance increased to values more than double that observed in both TPS-based CLs and Nafion-based electrodes at $20 \% \mathrm{RH}$. The large difference in membrane resistance for the TEBS-based electrode suggests insufficient water retention at low RHs resulting in a drier membrane that is less effective for the transport of proton to the cathode.

The decrease in performance in all regions (kinetic, ionic, and mass transport) when using the TEBS was unexpected since the theoretical benefits of adding a phenyl ring alluded to a more capacitive electrode, which should have similar or more enhanced electrode performance. Halfcell measurements also pointed to a more ionically conductive material with a lower electrode resistance. When the electrodes were compared it was evident that the TEBS-based electrode exhibited greater kinetic losses, which increased as the RH decreased. This was similar to what was observed with the Nafion-based electrode, except for the fact that the TEBS-based electrode requires a greater potential to drive the ORR forward. The increase in the required potential indicates that oxygen reduction is hindered in some way when using TEBS while this is not observed when using TPS and is not as excessive when using Nafion. Observations in literature indicate diffusion of oxygen is faster in hydrophilic domains, while solubility is higher in the hydrophobic domains of the ionomer[1,6]. When comparing the sulfonated organosilane materials it is evident that TEBS has a more hydrophobic structure because of the phenyl ring, and this can affect the oxygen diffusion and thus the oxygen reduction kinetics. With that being said, 
based off data observed in literature the higher hydrophobicity of TEBS will increase the solubility of oxygen in the ionomer when compared to TPS, but as a result decrease the oxygen diffusion to catalyst sites[1, 6,32$]$. Additionally, a low water content can also decrease the permeability of oxygen. Through EIS it was determined the TEBS-based electrode had the lowest water content observed by the large $\mathrm{R} \Sigma$ value. This low water content could be another reason to the large activation loss observed with the TEBS-based electrode. This increase in activation losses is also present for the Nafion-based electrode, but the water content was observed to be greater than TEBS through EIS which would allow sufficient permeability of oxygen. In the case of TPS, similar permeability to Nafion was observed between $80 \%$ and $60 \% \mathrm{RH}$, but due to the hygroscopic nature a higher water content is present at lower RH allowing better permeability than Nafion.

The replacement of TPS for TEBS has shown that the addition of a phenyl ring and shortening of the carbon chain has drastically decreased the performance in a fuel cell. The large increase in activation losses imply that there is a decrease in the oxygen reduction kinetics. The decrease in the oxygen reduction kinetics has been discussed by Peron et. al and was attributed to the increase in the hydrophobic region of the sulfonated side chain[33]. Holdcroft's group has also discussed the permeability of oxygen within the catalyst layer, and have suggested that the decrease of water content in an ionomer can change its morphology, leading to changes in the oxygen permeability[1]. That being said, if the total hydrophilicity of the organosilane can be increased this may increase the water retention within the electrode which may also increase the ORR kinetics.

The high electrode resistance observed with the TEBS-based electrode could indicate insufficient ion transport caused a decreased percolation pathway which may not allow ions to transport efficiently. On the other hand, the total silane loading may be too high causing the assumption that $\mathrm{R}_{\text {electronic }}<<\mathrm{R}_{\text {ionic }}$ to not be true. A high silane loading can increase Relectronic if there 
is a decrease in electronic conductivity by coverage of catalyst sites which has been seen to occur with high loadings of sPEEK in the catalyst layer[4].

\section{Conclusions}

A comparison of CCEs based on TPS and TEBS sulfonated silanes was performed. Electrochemical evaluation of the catalyst layers showed that TPS had a higher ECSA compared to TEBS. Performance tests showed that at high RH Nafion performed the best while both CCEs were prone to flooding. When compared in dry conditions TEBS-based electrode showed poor performance with high activation losses indicating poor oxygen reduction kinetics. The decreased kinetics was caused by poor water retention as indicated by EIS measurements. This was not observed for the TPS-based electrode, which was relatively insensitive to dry conditions. These results obtained have demonstrated that a small change to the sulfonated silane structure used in CCE fabrication can dramatically alter fuel cell performance characteristics. Further optimization of composition and/or synthetic methods could potentially be beneficial.

\section{Acknowledgements}

This work was supported by the Natural Sciences and Engineering Research Council (NSERC) of Canada, the Canada Foundation for Innovation, and Ontario Tech University. The authors thank Watlab at the University of Waterloo for collecting the TEM images.

\section{REFERENCES}

[1] S. Holdcroft, Fuel Cell Catalyst Layers: A Polymer Science Perspective, Chem. Mater., 26 (2014) 381-393.

[2] J. Larminie, A. Dicks, J. Larminie, A. Dicks, Fuel Cell Systems Explained, John Wiley \& Sons, Ltd, 2003. 
[3] Z. Qi, A. Kaufman, Improvement of water management by a microporous sublayer for PEM fuel cells, J. Power Sources, 109 (2002) 38-46.

[4] E.B. Easton, T.D. Astill, S. Holdcroft, Properties of Gas Diffusion Electrodes Containing Sulfonated Poly(ether ether ketone), J. Electrochem. Soc., 152 (2005) A752-A758.

[5] E.B. Easton, Z. Qi, A. Kaufman, P.G. Pickup, Chemical Modification of Proton Exchange Membrane Fuel Cell Catalysts with a Sulfonated Silane, Electrochem. Solid-State Lett., 4 (2001) A59-A61.

[6] J. Peron, Z. Shi, S. Holdcroft, Hydrocarbon proton conducting polymers for fuel cell catalyst layers, Energy \& Environmental Science, 4 (2011) 1575-1591.

[7] L. Li, J. Zhang, Y. Wang, Sulfonated poly(ether ether ketone) membranes for direct methanol fuel cell, Journal of Membrane Science, 226 159-167.

[8] N.N. Krishnan, H.J. Kim, J.H. Jang, S.Y. Lee, E. Cho, I.H. Oh, S.A. Hong, T.H. Lim, Sulfonated poly(ether sulfone)-based catalyst binder for a proton-exchange membrane fuel cell, J. Appl. Polym. Sci., 113 2499-2506.

[9] L. Rabinovich, O. Lev, Sol-Gel Derived Composite Ceramic Carbon Electrodes, Electroanalysis, 13 (2001) 265-275.

[10] M. Tsionsky, G. Gun, V. Glezer, O. Lev, Sol-Gel-Derived Ceramic-Carbon Composite Electrodes: Introduction and Scope of Applications, Anal. Chem., 66 (1994) 1747-1753.

[11] S. Ranganathan, E.B. Easton, Ceramic carbon electrode-based anodes for use in the $\mathrm{Cu}-\mathrm{Cl}$ thermochemical cycle, Int. J. Hydrogen Energy, 35 (2010) 4871-4876.

[12] J.I. Eastcott, E.B. Easton, Sulfonated silica-based fuel cell electrode structures for low humidity applications, J. Power Sources, 245 (2014) 487-494.

[13] N.E. De Almeida, E.B. Easton, Nafion/Sulfonated Silica Composite Membranes for PEM Fuel Cells, ECS Transactions, 28 (2010) 29-38.

[14] J.I. Eastcott, E.B. Easton, Electrochemical studies of ceramic carbon electrodes for fuel cell systems: A catalyst layer without sulfonic acid groups, Electrochimica Acta, 54 (2009) 3460-3466. [15] J.I. Eastcott, E.B. Easton, Sulfonated silica-based fuel cell electrode structures for low humidity applications, Journal of Power Sources, 245 (2014) 487-494.

[16] J.I. Eastcott, E.B. Easton, Investigation of Transport Mechanisms for Sulfonated Silica-Based Fuel Cell Electrode Structures, Journal of the Electrochemical Society, 162 (2015) F764-F771. 
[17] J.I. Eastcott, J.A. Powell, A.J. Vreugdenhil, E.B. Easton, Electrochemical and Morphological Studies of Ceramic Carbon Electrodes for Fuel Cell Systems, ECS Transactions, 41 (2011) 853864.

[18] J.I. Eastcott, K.M. Yarrow, A.W. Pedersen, E.B. Easton, Fuel cell electrode structures containing sulfonated organosilane-based proton conductors, Journal of Power Sources, 197 (2012) 102-106.

[19] Z.G. Qi, A. Kaufman, Improvement of water management by a microporous sublayer for PEM fuel cells, Journal of Power Sources, 109 (2002) 38-46.

[20] E.B. Easton, P.G. Pickup, An electrochemical impedance spectroscopy study of fuel cell electrodes, Electrochimica Acta, 50 (2005) 2469-2474.

[21] O.O. Reid, F.S. Saleh, E.B. Easton, Application of the Transmission Line EIS Model to Fuel Cell Catalyst Layer Durability, ECS Transactions, 61 (2014) 25-32.

[22] F.S. Saleh, E.B. Easton, Diagnosing Degradation within PEM Fuel Cell Catalyst Layers Using Electrochemical Impedance Spectroscopy, Journal of the Electrochemical Society, 159 (2012) B546-B553.

[23] M.C. Lefebvre, R.B. Martin, P.G. Pickup, Characterization of ionic conductivity profiles within proton exchange membrane fuel cell gas diffusion electrodes by impedance spectroscopy, Electrochemical and Solid State Letters, 2 (1999) 259-261.

[24] G.C. Li, P.G. Pickup, Ionic conductivity of PEMFC electrodes - Effect of Nafion loading, Journal of the Electrochemical Society, 150 (2003) C745-C752.

[25] M.L. Anderson, R.M. Stroud, D.R. Rolison, Enhancing the activity of fuel-cell reactions by designing three-dimensional nanostructured architectures: Catalyst-modified carbon-silica composite aerogels, Nano Letters, 2 (2002) 235-240.

[26] C.A. Morris, M.L. Anderson, R.M. Stroud, C.I. Merzbacher, D.R. Rolison, Silica sol as a nanoglue: Flexible synthesis of composite aerogels, Science, 284 (1999) 622-624.

[27] E.B. Easton, Z.G. Qi, A. Kaufman, P.G. Pickup, Chemical modification of proton exchange membrane fuel cell catalysts with a sulfonated silane, Electrochemical and Solid State Letters, 4 (2001) A59-A61.

[28] M. Atif, R. Bongiovanni, M. Giorcelli, E. Celasco, A. Tagliaferro, Modification and characterization of carbon black with mercaptopropyltrimethoxysilane, Applied Surface Science, 286 (2013) 142-148. 
[29] H. Yu, Y. Jin, Z. Li, F. Peng, H. Wang, Synthesis and characterization of sulfonated singlewalled carbon nanotubes and their performance as solid acid catalyst, Journal of Solid State Chemistry, 181 (2008) 432-438.

[30] C. Odetola, E.B. Easton, L. Trevani, Investigation of TiO2/carbon electrocatalyst supports prepared using glucose as a modifier, International Journal of Hydrogen Energy, 41 (2016) 81998208.

[31] S. Sharma, B.G. Pollet, Support materials for PEMFC and DMFC electrocatalysts-A review, Journal of Power Sources, 208 (2012) 96-119.

[32] F.N. Büchi, M. Wakizoe, S. Srinivasan, Microelectrode Investigation of Oxygen Permeation in Perfluorinated Proton Exchange Membranes with Different Equivalent Weights, J. Electrochem. Soc., 143 (1996) 927-932.

[33] J. Peron, D. Edwards, A. Besson, Z. Shi, S. Holdcroft, Microstructure-Performance Relationships of sPEEK-Based Catalyst Layers, J. Electrochem. Soc., 157 (2010) B1230-B1236. 